\title{
Lax type formulas with lower semicontinuous initial data and hypercontractivity results
}

\author{
Antonio Avantaggiati and Paola Loreti
}

\begin{abstract}
In Avantaggiati and Loreti [Ric. Mat. 57(2):171-202, 2008] we studied the Cauchy problem for a class of Hamilton-Jacobi equations with initial data verifying the Lipschitz condition. In this paper we extend those results to the case in which the initial data are lower semicontinuous [in the following lsc], and are lower bounded and semiconvex. Here we prove hypercontractivity results and new Logarithm Sobolev Inequalities (shortly, LSI).
\end{abstract}

Mathematics Subject Classification. Primary 35F25, 49L25;

Secondary 26D15.

Keywords. Hopf-Lax formulas, Hamilton-Jacobi equations, Functional inequalities.

\section{Introduction}

We consider the Cauchy problem

$$
\begin{cases}u_{t}(x, t)+\alpha x D u(x, t)+H(D u(x, t))=0, & (x, t) \in \mathbb{R}^{n} \times \mathbb{R}_{+}, \\ u(x, 0)=u_{0}(x) & x \in \mathbb{R}^{n},\end{cases}
$$

where $\alpha$ is a positive real number and $H \in C^{1}\left(\mathbb{R}^{n}\right)$, convex, $p$-homogeneous, and even. In the previous paper [2] we studied the problem (1.1) taking $H(x)=$ $\frac{1}{2}|x|^{2}$ and $n=1$, in [4] we considered the problem for more general $H$; in the paper [3] we have set $\sum_{j} \alpha_{j} x_{j} \partial_{x_{j}} u(x, t)$ instead of $\alpha x D u(x, t)$, and we studied the case

$$
\begin{cases}u_{t}(x, t)+\sum_{j} \alpha_{j} x_{j} \partial_{x_{j}} u(x, t)+\frac{1}{2}|D u(x)|^{2}=0, & (x, t) \in \mathbb{R}^{n} \times \mathbb{R}_{+}, \\ u(x, 0)=u_{0}(x) & x \in \mathbb{R}^{n} .\end{cases}
$$

In the papers [2-4] we assumed that $u_{0}$ is Lipschtz continuous in all the space $\mathbb{R}^{n}$ and admissible, which means

$$
\int_{\mathbb{R}^{n}} \exp \left[u_{0}(x)\right] d x<+\infty .
$$


The assumption (1.3) is mainly used to prove LSI and it appears in several papers in the literature. For an ealier study of LSI in connection with Hamilton-Jacobi equations we refer to the papers $[5,11,12,14]$.

In this paper, we extend some of the results of the papers [2-4], and we explain here, shortly, the content of it.

In Sect. 2 we adapt the definition of lower boundednes [see definition (2.1)]. This definition was suggested by the estimates obtained in our previuos papers.

The goodness of such definition is proved in the same section. In fact, if $u_{0}$ is lsc and so lower bounded, the function

$$
\left(Q_{t} u_{0}\right)(x)=\inf _{y \in \mathbb{R}^{n}}\left(u_{0}(y)+\left(\frac{\alpha p}{1-e^{-2 \alpha p t}}\right)^{q-1} H^{*}\left(y-e^{-\alpha t} x\right)\right)
$$

attains the minimum [see theorem (2.2)]. Furthermore, we prove that $\left(Q_{t} u_{0}\right)(x)$ is lsc and it is viscosity solution of (1.1); see the theorem 2.4 and the propositions 2.7 , and 2.8 .

In the next sections, we are concerned with the hypercontractivity and the Logarithmic Sobolev Inequalities and we are mainly interested to improve the results of [3].

In such sections we make on $u_{0}$ a particular type of semiconvexity hypothesis. In Sect. 3, under such hypothesis, we represent the solution of the problem (1.2) as difference of two functions, one of which solution of the Hamilton-Jacobi equation, more precisely as the difference of a $C^{\infty}$ function and a function with Lipschitz continuous gradient; see Theorem 3.6.

In Sects. 4 and 5, we replace the assumption of admissibility assuming that $u_{0}$ has the behaviour

$$
u_{0}(x)=\mathrm{o}(H(x)), \quad \text { for }|x| \rightarrow+\infty .
$$

With such framework we get new hypercontractivity weighted estimates and Logarithmic Sobolev Inequalities, which extend those of papers [3]. In Theorems 4.4, 4.6, 5.1 and 5.3 some new results are stated and proved.

\section{Problem and main assumptions}

The asssumptions used in the paper on the function $H$ are

$$
\begin{array}{r}
H \text { is } \mathrm{C}^{1}\left(\mathbb{R}^{\mathrm{n}}\right), H \text { is an even, nonnegative, convex function, } \\
\text { positive - homogeneous of degree } p>1
\end{array}
$$

We recall that $H^{\star}$ denote the conjugate of $H$, i.e. its Legendre transform, defined by

$$
H^{\star}(\zeta)=\sup _{y \in \mathbb{R}^{n}}\{y \zeta-H(y)\} \quad \zeta \in \mathbb{R}^{n}
$$


As well known, $H^{\star}$ is an even, non negative, and convex function, positively homogeneous of degree $q$, with $\frac{1}{p}+\frac{1}{q}=1$.. We consider

$$
u(x, t)=\inf _{y \in \mathbb{R}^{n}}\left(u_{0}\left(e^{-\alpha t} x-\frac{1-e^{-\alpha p t}}{\alpha p} y\right)+\frac{1-e^{-\alpha p t}}{\alpha p} H^{\star}(y)\right),
$$

or the equivalent formula,

$$
u(x, t)=\inf _{y \in \mathbb{R}^{n}}\left(u_{0}(y)+\left(\frac{\alpha p}{1-e^{-\alpha p t}}\right)^{q-1} H^{\star}\left(y-e^{-\alpha t} x\right)\right) .
$$

We adapt the definition of lower boundednes to our problem

Definition 2.1. We say that $u_{0}$ is $\left(H^{\star}, \alpha\right)$ lower bounded if there exist $\left.\left.A \in\right] 0, \alpha\right]$ and $x_{0} \in \mathbb{R}^{n}$, such that

$$
u_{0}(x) \geq-(A p)^{q-1} H^{\star}\left(x-x_{0}\right) .
$$

The asssumption on the function $u_{0}$ is

$u_{0}$ is real valued functions lsc on all the space $\mathbb{R}^{n},\left(H^{\star}, \alpha\right)$ lower bounded.

\subsection{Regularity properties of $u$}

We have the result

Theorem 2.2. If (2.1), (2.4) hold, then the function $u$, defined by (2.3), has the following property

$$
\begin{aligned}
& \left.\forall x \in \mathbb{R}^{n} \forall t \in\right] 0,+\infty\left[, \exists y_{*} \in \mathbb{R}^{n}\right. \text { s.t. } \\
& u(x, t)=u_{0}\left(y_{*}\right)+\left(\frac{\alpha p}{1-e^{-\alpha p t}}\right)^{q-1} H^{\star}\left(y_{*}-e^{-\alpha t} x\right) .
\end{aligned}
$$

Proof. Let $x, t$ fixed; first of all, we observe that

$$
u(x, t) \leq u_{0}\left(e^{-\alpha t} x\right) .
$$

On the other hand, from the hypothesis, since $0<A \leq \alpha$, we get

$$
\lim _{y \rightarrow \infty}\left[-(A p)^{q-1} H^{\star}\left(y-x_{0}\right)+\left(\frac{\alpha p}{1-e^{-\alpha p t}}\right)^{q-1} H^{\star}\left(y-e^{-\alpha t} x\right)\right]=+\infty .
$$

Thanks to (2.4) it is possible therefore to find $R$ such that

$$
|y|>R \Rightarrow u_{0}(y)+\left(\frac{\alpha p}{1-e^{-\alpha p t}}\right)^{q-1} H^{\star}\left(y-e^{-\alpha t} x\right)>u_{0}\left(e^{-\alpha t} x\right) .
$$

It follows that the function $U_{0}$ defined by:

$$
U_{0}(y)=u_{0}(y)+\left(\frac{\alpha p}{1-e^{-\alpha p t}}\right)^{q-1} H^{\star}\left(y-e^{-\alpha t} x\right),
$$

is lower bounded on the ball $B_{R}=\left\{y \in \mathbb{R}^{n}\right.$ s.t. $\left.|y| \leq R\right\}$, and the set $E$ of the points $y \in \mathbb{R}^{N}$, on which it must be computed the infimum of $U_{0}(y)$, may be $B_{R}$. Since $U_{0}(y)$ is lsc and $B_{R}$ is a compact set, the thesis follows with $y_{\star} \in B_{R}$. 
Remark 2.3. If the assumptions of the definition (2.1) hold with $A>\alpha$, then there exists $t \in] 0,+\infty[$ such that

$$
\frac{\alpha}{1-e^{-\alpha p t}}<A
$$

and the function $U_{0}$ [defined by (2.5)] may be lower unbounded. Therefore the thesis of the theorem (2.2) may be false. It is useful to observe that (2.6) is equivalent, for $A>\alpha$, to $t>\frac{1}{\alpha p} \log \frac{A}{A-\alpha}$.

Our purpose is now the following

Theorem 2.4. If (2.1), (2.4) hold, then the function $u$, defined by (2.3) is lsc on $\left.\mathbb{R}^{n} \times\right] 0,+\infty[$.

Proof. It is enough to state that all the sub-level set of $u(x, t)$ are closed in $\left.\mathbb{R}^{n} \times\right] 0,+\infty[$. Let

$$
S_{r}(u)=\left\{(x, t) \in \mathbb{R}^{n} \times\right] 0,+\infty[\text { s.t. } u(x, t) \leq r\},
$$

with $r \in \mathbb{R}$, such that $S_{r}(u) \neq \emptyset$. We have to prove that $S_{r}(u)$ is closed. Let us consider a sequence $\left(x_{k}, t_{k}\right)$ such that

$$
u\left(x_{k}, t_{k}\right) \leq r \quad \text { for all } k \in \mathbb{N} ; x_{k} \rightarrow x \text { and } t_{k} \rightarrow t .
$$

For each $k \in \mathbb{N}$ there exists $y_{k} \in \mathbb{R}^{n}$ such that

$$
u\left(x_{k}, t_{k}\right)=u_{0}\left(y_{k}\right)+\left(\frac{\alpha p}{1-e^{-\alpha p t_{k}}}\right)^{q-1} H^{\star}\left(y_{k}-e^{-\alpha t_{k}} x_{k}\right) .
$$

As first, we prove that the sequence $\left(y_{k}\right)$ is bounded.

We argue by contradiction. Assume $\left(y_{k}\right)$ unbounded. Without restricting the generality, we may assume that

$$
\lim _{k \rightarrow \infty}\left|y_{k}\right|=+\infty
$$

From (2.7) it follows

$$
\begin{aligned}
r & \geq u\left(x_{k}, t_{k}\right)=u_{0}\left(y_{k}\right)+\left(\frac{\alpha p}{1-e^{-\alpha p t_{k}}}\right)^{q-1} H^{\star}\left(y_{k}-e^{-\alpha t_{k}} x_{k}\right) \\
& \geq-(A p)^{q-1} H^{\star}\left(y_{k}-x_{0}\right)+\left(\frac{\alpha p}{1-e^{-\alpha p t_{k}}}\right)^{q-1} H^{\star}\left(y_{k}-e^{-\alpha t_{k}} x_{k}\right) .
\end{aligned}
$$

Since $e^{-\alpha p t_{k}} \rightarrow e^{-\alpha p t}, x_{k} \rightarrow x$ and

$$
(A p)^{q-1}<\left(\frac{\alpha p}{1-e^{-\alpha p t_{k}}}\right)^{q-1}
$$

we obtain

$$
\lim _{k \rightarrow \infty}-(A p)^{q-1} H^{\star}\left(y_{k}-x_{0}\right)+\left(\frac{\alpha p}{1-e^{-\alpha p t_{k}}}\right)^{q-1} H^{\star}\left(y_{k}-e^{-\alpha t_{k}} x_{k}\right)=+\infty
$$

which is impossible by (2.7). Therefore $\left(y_{k}\right)$ is bounded. Then there exists a convergent subsequence. We may assume that

$$
\exists y_{0} \in \mathbb{R}^{n} \text { such that } \lim _{k \rightarrow \infty} y_{k}=y_{0} \text {. }
$$


From (2.8) and (2.9) we get

$$
\begin{aligned}
r & \geq \liminf _{k} u\left(x_{k}, t_{k}\right)=\liminf _{k}\left[u_{0}\left(y_{k}\right)+\left(\frac{\alpha p}{1-e^{-\alpha p t_{k}}}\right)^{q-1} H^{\star}\left(y_{k}-e^{-\alpha t_{k}} x_{k}\right)\right] \\
& \left.\geq u_{0}\left(y_{0}\right)+\left(\frac{\alpha p}{1-e^{-\alpha p t}}\right)^{q-1} H^{\star}\left(y_{0}-e^{-\alpha t} x\right)\right] \geq u(x, t)
\end{aligned}
$$

and this complete the proof.

We have to prove now that $u(x, t)$, for $t \rightarrow 0$, takes the initial datum $u_{0}$. Actually the meaning of such assertion is given by the following

Theorem 2.5. If (2.1), (2.4) hold, then for every $x^{\prime} \in \mathbb{R}^{n}$

$$
\lim _{r \rightarrow 0} \inf _{(y, s)}\left\{u(y, s) ; \quad\left|y-x^{\prime}\right|<r, 0<s<r\right\}=u_{0}\left(x^{\prime}\right) .
$$

Proof. We fix $x^{\prime} \in \mathbb{R}^{n}$, and we introduce the function

$$
v(x, t, y)=\varphi(t) H^{*}\left(\frac{y-e^{-\alpha t} x}{\varphi(t)}\right), \quad \text { where } \varphi(t)=\frac{1-e^{-\alpha p t}}{\alpha p}, \quad t \in[0,+\infty[\text {. }
$$

Such function $v$ is convex with respect to both the variables $x, y$. On the other hand we know that for every $(x, t) \in \mathbb{R}^{n} \times \mathbb{R}_{+}$there exists $y_{*} \in \mathbb{R}^{n}$ such that $u(x, t)=u_{0}\left(y_{*}\right)+v\left(x, t, y_{*}\right) \geq u_{0}\left(y_{*}\right)+s\left(y_{*}-e^{-\alpha t} x\right)-\varphi(t) H(s), \quad \forall s \in \mathbb{R}^{n}$. We define

$$
s(r)=\frac{r}{q}\left(D H^{*}\right)\left(y_{*}-e^{-\alpha t} x\right),
$$

and observe, using $s(r)$, the lower estimation of $u_{0}$, the q-homogenity and the convexity of $H^{*}$, that

$$
\begin{aligned}
& u(x, t) \geq-(A p)^{q-1} H^{*}\left(y_{*}-x_{0}\right)+r H^{*}\left(y_{*}-e^{-\alpha t} x\right)-\varphi(t) H(s(r)) \\
& \quad \geq\left(r-(2 A p)^{q-1}\right) H^{*}\left(y_{*}-e^{-\alpha t} x\right)-(2 A p)^{q-1} H^{*}\left(e^{-\alpha t} x-x_{0}\right)-\varphi(t) H(s(r))
\end{aligned}
$$

We fix $\epsilon$, and find $\delta$ so that

$$
\left|y-x^{\prime}\right|<4 \delta \Rightarrow u_{0}(y)>u_{0}\left(x^{\prime}\right)-\epsilon .
$$

We introduce the set

$$
\mathcal{E}=B\left(x^{\prime}, \delta\right) \times(0, \tau)
$$

We now observe that, when $(x, t) \in \mathcal{E}$, e.g., $(x, t)$ is in a bounded set, also $y_{*}$ is in a bounded set, then there exists $R(\mathcal{E}) \in \mathbb{R}_{+}$such that

$$
\left|y_{*}\right| \leq R(\mathcal{E}), \forall(x, t) \in \mathcal{E} .
$$

For $(x, t) \in \mathcal{E}$ and $\tau$ small enough,

$$
\left|e^{-\alpha t} x-x^{\prime}\right|=\left|e^{-\alpha t} x-x\right|+\left|x-x^{\prime}\right| \leq\left(1-e^{-\alpha t}\right)|x|+\delta<2 \delta,
$$

so that $e^{-\alpha t} x \in B\left(x^{\prime}, 2 \delta\right)$. It follows that $\forall(x, t) \in \mathcal{E}$

$$
\left|y_{*}-x^{\prime}\right|>4 \delta \Rightarrow\left|y_{*}-e^{-\alpha t} x\right|>2 \delta .
$$

As consequence there exists a constant $c_{1}$ such that

$$
H^{*}\left(y_{*}-e^{-\alpha t} x\right) \geq c_{1}>0, \quad \forall(x, t) \in \mathcal{E}, \quad\left|y_{*}-x^{\prime}\right|>4 \delta
$$


and (2.10) implies for some positive constant $c_{2}$ and $c_{3}$

$$
\begin{aligned}
\left|\frac{1}{q}\left(D H^{*}\right)\left(y_{*}-e^{-\alpha t} x\right)\right| & \leq c_{2}, \\
H^{*}\left(e^{-\alpha t} x-x_{0}\right) & \leq c_{3}
\end{aligned}
$$

We fix $r \in \mathbb{R}_{+}$such that

$$
\left(r-(2 A p)^{q-1}\right) c_{1}-(2 A p)^{q-1} c_{3}>u_{0}\left(x^{\prime}\right),
$$

then, $\forall(x, t) \in \mathcal{E}$, and $\left|y_{*}-x^{\prime}\right|>4 \delta$,

$$
\begin{aligned}
& \left(r-(2 A p)^{q-1}\right) H^{*}\left(y_{*}-e^{-\alpha t} x\right)-(2 A p)^{q-1} H^{*}\left(e^{-\alpha t} x-x_{0}\right) \\
& \quad \geq\left(r-(2 A p)^{q-1}\right) c_{1}-(2 A p)^{q-1} c_{3}>u_{0}\left(x^{\prime}\right) .
\end{aligned}
$$

Next, we choose $\tau$ so that, for $r$ verifying $\left(r-(2 A p)^{q-1}\right) c_{1}-(2 A p)^{q-1} c_{3}>$ $u_{0}\left(x^{\prime}\right)$,

$$
\varphi(\tau) \max _{|s| \leq r c_{2}} H(s) \leq \epsilon
$$

and we get

$$
\left(r-(2 A p)^{q-1}\right) H^{*}\left(y_{*}-e^{-\alpha t} x\right)-(2 A p)^{q-1} H^{*}\left(e^{-\alpha t} x-x_{0}\right)-\varphi(t) H(s) \geq u_{0}\left(x^{\prime}\right)-\epsilon .
$$

Set $m=\min \left\{m_{1}, m_{2}\right\}$, with

$$
\begin{aligned}
m_{1}= & \inf _{\left|y_{*}-x^{\prime}\right|>4 \delta}\left(u_{0}\left(y_{*}\right)+r H^{*}\left(y_{*}-e^{-\alpha t} x\right)-\varphi(t) H(s(r))\right) \\
\geq & \inf _{\left|y_{*}-x^{\prime}\right|>4 \delta}\left(\left(r-(2 A p)^{q-1}\right) H^{*}\left(y_{*}-e^{-\alpha t} x\right)-(2 A p)^{q-1} H^{*}\left(e^{-\alpha t} x-x_{0}\right)\right. \\
& \quad-\varphi(t) H(s(r))) \geq u_{0}\left(x^{\prime}\right)-\epsilon
\end{aligned}
$$

and

$$
\begin{aligned}
m_{2} & =\inf _{\left|y_{*}-x^{\prime}\right| \leq 4 \delta}\left(u_{0}\left(y_{*}\right)+r H^{*}\left(y_{*}-e^{-\alpha t} x\right)-\varphi(t) H(s(r))\right) \\
& \geq \inf _{\left|y_{*}-x^{\prime}\right| \leq 4 \delta}\left(u_{0}\left(y_{*}\right)-\varphi(t) H(s(r))\right) \geq u_{0}\left(x^{\prime}\right)-2 \epsilon .
\end{aligned}
$$

Since

$$
u(x, t) \geq m
$$

we deduce that

$$
\lim _{r \rightarrow 0} \inf _{(y, s)}\left\{u(y, s) ; \quad\left|y-x^{\prime}\right|<r, 0<s<r\right\} \geq u_{0}\left(x^{\prime}\right) .
$$

On the other hand

$$
u(x, t) \leq u_{0}\left(e^{-\alpha t} x\right),
$$

for any $(x, t) \in \mathcal{E}$, and by the lsc of $u_{0}$, we deduce that

$$
\lim _{r \rightarrow 0} \inf _{(y, s)}\left\{u(y, s) ; \quad\left|y-x^{\prime}\right|<r, 0<s<r\right\} \leq u_{0}\left(x^{\prime}\right),
$$

and the result follows. 
Next we observe that

$$
\left(Q_{s} u_{0}\right)(x)=\min _{w}\left\{u_{0}\left[e^{-\alpha s} x+\left(\frac{1-e^{-\alpha p s}}{\alpha p}\right)^{\frac{1}{p}} w\right]+H^{\star}(w)\right\} .
$$

We can conclude this section with

Theorem 2.6. If $u_{0}$ is lsc and $H$ is convex, even, $p$-homogeneous with $p>1$, then

$$
Q_{t}\left(Q_{s}\right)\left(u_{0}\right)=Q_{t+s}\left(u_{0}\right) \quad \forall t, s \in \mathbb{R}_{+} .
$$

Proof. We give here a short sketch of the proof (see [4]). By

$$
\begin{aligned}
Q_{t}\left(Q_{s}\right)(x)= & \min _{z}\left\{\operatorname { m i n } _ { w } \left\{u _ { 0 } \left[e^{-\alpha s}\left[e^{-\alpha t} x+\left(\frac{1-e^{-\alpha p t}}{\alpha p}\right)^{\frac{1}{p}} z\right]\right.\right.\right. \\
& \left.\left.\left.+\left(\frac{1-e^{-\alpha p s}}{\alpha p}\right)^{\frac{1}{p}} w\right]+H^{\star}(w)\right\}+H^{\star}(z)\right\} \\
= & \min _{(z, w)}\left\{u_{0}\left[e^{-\alpha(t+s)} x+e^{-\alpha s}\left(\frac{1-e^{-\alpha p t}}{\alpha p}\right)^{\frac{1}{p}} z+\left(\frac{1-e^{-\alpha p s}}{\alpha p}\right)^{\frac{1}{p}} w\right]\right. \\
& \left.+H^{\star}(w)+H^{\star}(z)\right\} .
\end{aligned}
$$

We make the change of variables

$$
\left\{\begin{array}{l}
e^{-\alpha s}\left(\frac{1-e^{-\alpha p t}}{\alpha p}\right)^{\frac{1}{p}} z+\left(\frac{1-e^{-\alpha p s}}{\alpha p}\right)^{\frac{1}{p}} w=\left(\frac{1-e^{-\alpha p(t+s)}}{p \alpha}\right)^{\frac{1}{p}} u \\
-\left(\frac{1-e^{-\alpha p s}}{\alpha p}\right)^{\frac{1}{q}} z+\left(\frac{1-e^{-\alpha p t}}{\alpha p}\right)^{\frac{1}{q}} e^{-\alpha(p-1) s} w=\left(\frac{1-e^{-\alpha(t+s) p}}{\alpha p}\right)^{\frac{1}{q}} v .
\end{array}\right.
$$

We put

$$
A=e^{-\alpha p s}\left(\frac{1-e^{-\alpha p t}}{\alpha p}\right) ; \quad B=\frac{1-e^{-\alpha p s}}{\alpha p} .
$$

We observe that

$$
A+B=\frac{1-e^{-\alpha p(t+s)}}{\alpha p}
$$

Introducing

$$
\lambda=\frac{A}{A+B}, \quad 1-\lambda=\frac{B}{A+B},
$$

the previous transformation may be written

$$
\left\{\begin{array}{l}
\lambda^{\frac{1}{p}} z+(1-\lambda)^{\frac{1}{p}} w=u \\
-(1-\lambda)^{\frac{1}{q}} z+\lambda^{\frac{1}{q}} w=v
\end{array}\right.
$$

whose inverse is given by

$$
\left\{\begin{array}{l}
\lambda^{\frac{1}{q}} u-(1-\lambda)^{\frac{1}{p}} v=z \\
(1-\lambda)^{\frac{1}{q}} u+\lambda^{\frac{1}{p}} v=w
\end{array}\right.
$$


From (2.12), it results

$$
\begin{aligned}
Q_{t}\left(Q_{s} u_{0}\right)(x)= & \min _{u}\left\{u_{0}\left[e^{-\alpha(t+s)} x+\left(\frac{1-e^{-\alpha p(t+s)}}{\alpha p}\right)^{\frac{1}{p}} u\right]\right. \\
& \left.+\min _{v}\left[H^{\star}\left(\lambda^{\frac{1}{q}} u-(1-\lambda)^{\frac{1}{p}} v\right)+H^{\star}\left((1-\lambda)^{\frac{1}{q}} u+\lambda^{\frac{1}{p}} v\right)\right]\right\}
\end{aligned}
$$

By an easy computation, using the convexity of $H^{\star}$, we have

$$
\min _{v}\left[H^{\star}\left(\lambda^{\frac{1}{q}} u-(1-\lambda)^{\frac{1}{p}} v\right)+H^{\star}\left((1-\lambda)^{\frac{1}{q}} u+\lambda^{\frac{1}{p}} v\right)\right]=H^{\star}(u) .
$$

Then from (2.12), (2.13) the thesis follows.

\subsection{Viscosity solutions}

In this subsection we show that $u(x, t)$ defined by $(2.2)$, is a viscosity solution of the problem (1.1). For definition and main properties of viscosity solutions we refere to [7], [8] and, as reference closer to our case, to $[9,10]$.

Proposition 2.7. Assume (2.1), (2.4). Then u defined by (2.2) is a viscosity subsolution of (1.1).

Proof. Given a $C^{1}$ test function $\varphi(x, t)$, let us assume $u-\varphi$ has maximum relative point $\left(x_{0}, t_{0}\right)$, and without leading the generality, we may assume

$$
u\left(x_{0}, t_{0}\right)=\varphi\left(x_{0}, t_{0}\right) .
$$

Using the semigroup property of $Q_{t}$, we have

$$
Q_{s}\left(Q_{t_{0}-s} u_{0}\right)\left(x_{0}\right)=Q_{t_{0}} u_{0}\left(x_{0}\right)
$$

In explicit form

$$
\begin{aligned}
& u\left(x_{0}, t_{0}\right)=Q_{s} u\left(x_{0}, t_{0}-s\right) \\
& \quad=\min _{y}\left\{u\left(e^{-\alpha s} x_{0}+\frac{1-e^{-\alpha p s}}{\alpha p} y, t_{0}-s\right)+\frac{1-e^{-\alpha p s}}{\alpha p} H^{\star}(y)\right\} .
\end{aligned}
$$

We fix a neighborhood $I_{0}$ of $\left(x_{0}, t_{0}\right)$ such that

$$
u(x, t)-\varphi(x, t) \leq 0 \quad \forall(x, t) \in I_{0} .
$$

From (2.14), for any fixed $y \in R^{n}$ we have

$$
\varphi\left(x_{0}, t_{0}\right)=u\left(x_{0}, t_{0}\right) \leq u\left(e^{-\alpha s} x_{0}+\frac{1-e^{-\alpha p s}}{\alpha p} y, t_{0}-s\right)+\frac{1-e^{-\alpha p s}}{\alpha p} H^{\star}(y) .
$$

We can find $\delta>0$ such that $\forall s \in] 0, \delta[$ :

$$
\left(e^{-\alpha s} x_{0}+\frac{1-e^{-\alpha p s}}{\alpha p} y, t_{0}-s\right) \in I_{0} .
$$

Therefore by (2.15) we get

$$
\begin{aligned}
& \varphi\left(x_{0}, t_{0}\right)=u\left(x_{0}, t_{0}\right) \\
& \quad \leq \varphi\left(e^{-\alpha s} x_{0}+\frac{1-e^{-\alpha p s}}{\alpha p} y, t_{0}-s\right)+\frac{1-e^{-\alpha p s}}{\alpha p} H^{\star}(y), \quad \forall s \in[0, \delta] .
\end{aligned}
$$


Then the function

$$
[0, \delta] \ni s \rightarrow \varphi\left(e^{-\alpha s} x_{0}+\frac{1-e^{-\alpha p s}}{\alpha p} y, t_{0}-s\right)+\frac{1-e^{-\alpha p s}}{\alpha p} H^{\star}(y)=: \psi(s),
$$

has a minimum at $s=0$. Therefore we have $\psi^{\prime}(0) \geq 0$, that is

$$
\left(-\alpha x_{0}+y\right) \cdot D \varphi\left(x_{0}, t_{0}\right)-\varphi_{t}\left(x_{0}, t_{0}\right)+H^{\star}(y) \geq 0
$$

or, equivalently

$$
\varphi_{t}\left(x_{0}, t_{0}\right)+\alpha x_{0} D \varphi\left(x_{0}, t_{0}\right)+y\left(-D \varphi\left(x_{0}, t_{0}\right)\right)-H^{\star}(y) \leq 0 .
$$

Since this inequality is true for all $y \in \mathbb{R}^{n}$ we obtain that $u(x, t)$ is viscosity subsolution of the Hamilton-Jacobi equation (1.1).

We can prove that $u(x, t)$ is a viscosity supersolution of (1.1), if the lsc datum $u_{0}$ has an approximation property (see [9] where such property is used), as it is shown in the following

Theorem 2.8. Assume (2.1), (2.4). If there exists a family of $C^{1}$ functions: $\left(u_{\epsilon}(x)\right)_{\epsilon \in] 0,1]}$ such that

$$
u_{0}(x)=\inf _{\epsilon \in] 0,1]} u_{\epsilon}(x) \quad \forall x \in \mathbb{R}^{n},
$$

then $u(x, t)$ defined by $(2.2)$ is a viscosity supersolution of (1.1)

Proof. We observe, by (2.2), that

$$
\begin{aligned}
u(x, t) & =\inf _{y}\left(u_{0}\left(e^{-\alpha t} x-\frac{1-e^{-\alpha p t}}{\alpha p} y\right)+\frac{1-e^{-\alpha p t}}{\alpha p} H^{\star}(y)\right) \\
& =\inf _{(y, \epsilon)}\left\{u_{\epsilon}\left(e^{-\alpha t} x-\frac{1-e^{-\alpha p t}}{\alpha p} y\right)+\frac{1-e^{-\alpha p t}}{\alpha p} H^{\star}(y)\right\} .
\end{aligned}
$$

Let us consider the $C^{1}\left(\mathbb{R}^{n} \times \mathbb{R}_{+}\right)$functions

$$
u^{\epsilon}(x, t ; y)=u_{\epsilon}\left(e^{-\alpha t} x-\frac{1-e^{-\alpha p t}}{\alpha p} y\right)+\frac{1-e^{-\alpha p t}}{\alpha p} H^{\star}(y)
$$

and we observe that

$$
\begin{aligned}
u_{t}^{\epsilon}(x, t ; y) & =\left(-\alpha e^{-\alpha t} x-e^{-\alpha p t} y\right) D u_{\epsilon}\left(e^{-\alpha t} x-\frac{1-e^{-\alpha p t}}{\alpha p} y\right)+e^{-\alpha p t} H^{\star}(y) \\
D u^{\epsilon}(x, t ; y) & =e^{-\alpha t} D u_{\epsilon}\left(e^{-\alpha t} x-\frac{1-e^{-\alpha p t}}{\alpha p} y\right) ; \\
H\left(D u^{\epsilon}(x, t ; y)\right) & =e^{-\alpha p t} H\left(D u_{\epsilon}\left(e^{-\alpha t} x-\frac{1-e^{-\alpha p t}}{\alpha p} y\right)\right) .
\end{aligned}
$$

It follows

$$
\begin{aligned}
u_{t}^{\epsilon}(x, t ; y) & =-\alpha x D u^{\epsilon}(x, t ; y)-e^{-\alpha p t}\left[y D u_{\epsilon}\left(e^{-\alpha t} x-\frac{1-e^{-\alpha p t}}{\alpha p} y\right)-H^{\star}(y)\right] \\
& \geq-\alpha x D u^{\epsilon}(x, t ; y)-e^{-\alpha p t} H\left(D u_{\epsilon}\left(e^{-\alpha t} x-\frac{1-e^{-\alpha p t}}{\alpha p} y\right)\right) \\
& =-\alpha x D u^{\epsilon}(x, t ; y)-H\left(D u^{\epsilon}(x, t ; y)\right) .
\end{aligned}
$$


Therefore we have, for every $y \in R^{n}$ and $\epsilon \in(0, \delta)$ :

$$
u_{t}^{\epsilon}(x, t ; y)+\alpha x D u^{\epsilon}(x, t ; y)+H\left(D u^{\epsilon}(x, t ; y)\right) \geq 0 .
$$

We get that, for any $y$, and $\epsilon \in(0,1], u^{\epsilon}(x, t ; y)$ is a viscosity supersolution of (1.1). Then the result follows.

Hence $u$, under the assumptions of the theorem (2.8), is a viscosity solution of the problem (1.1).

\section{Complementary results and assumptions}

First of all we wish to show some simple properties of the semigroup $t \rightarrow Q_{t}$, with respect to initial datum $u_{0}$. It is a kind of monotonicity. Given two initial data $u_{1}$ and $u_{2}$, it is easy to prove the implication

$$
u_{1} \leq u_{2} \Rightarrow\left(Q_{t} u_{1}\right) \leq\left(Q_{t} u_{2}\right) .
$$

Keeping in mind the condition of lower boundedness imposed to the initial datum $u_{0}$, i.e.

$$
u_{0}(x) \geq-(A p)^{q-1} H^{\star}\left(x-x_{0}\right),
$$

for every $x \in \mathbb{R}^{n}$, and $A \leq \alpha$ and $x_{0}$ is a fixed point of $\mathbb{R}^{n}$. By the monotonicity property (3.1), we get

$$
u(x, t) \geq Q_{t}\left(-(A p)^{q-1} H^{\star}\left(x-x_{0}\right)\right) .
$$

By an easy computation, we get

$$
u(x, t) \geq-\left(\frac{A p \alpha}{\alpha-A\left(1-e^{-\alpha p t}\right)}\right)^{q-1} H^{\star}\left(e^{-\alpha t} x-x_{0}\right) .
$$

We point out that the right hand side is the regular solution

$$
Q_{t}\left(-(A p)^{q-1} H^{*}(x-\bar{x})\right)=u^{*}(x, t),
$$

which in [4], verified equality and show the optimality of hypercontractivity and ultracontractivity inequalities.

As general reference to the following notions see $[1,6,13]$.

We now recall the definition of semiconvex function.

Definition 3.1. $f: \mathbb{R} \rightarrow \mathbb{R} \cup\{+\infty\}$ is semiconvex, or convex up to a square, if there exists some constant $c \geq 0$ such that

$$
f(x)+c|x|^{2}
$$

is convex. 
3.1. The particular case $H(x)=\frac{1}{2}|x|^{2}$

We are concerned, in this subsection, with the Hamilton-Jacobi equation

$$
u_{t}(x, t)+\sum_{i=1}^{n} \alpha_{i} x_{i} \partial_{x_{i}} u(x, t)+\frac{1}{2} \sum_{i=1}^{n}\left(\partial_{x_{i}}(x, t)\right)^{2}=0
$$

where all the $\alpha_{i}$ are positive real numbers, and we put $\alpha=\left(\alpha_{1}, \ldots, \alpha_{n}\right)$. Next we adapt the definition of $\left(H^{\star}, \alpha\right)$ lower boundedness and we give a notion a semiconvexity connected to the class of Hamilton-Jacobi equation we are considering.

Definition 3.2. We say that $u_{0}$ is $\alpha$ lower bounded if

$$
\left.\exists B \in] 0, \max _{i=1, \ldots, n} \alpha_{i}\right] \quad \text { and } x_{0} \in \mathbb{R}^{n},
$$

such that

$$
u_{0}(x) \geq-B\left|x-x_{0}\right|^{2} .
$$

Definition 3.3. Let $u_{0}$ a real valued function, we say that $u_{0}$ is $\alpha$-semiconvex, [strictly $\alpha$-semiconvex], if $\exists A_{j} \in \mathbb{R}_{+}, A_{j} \leq \alpha_{j},\left[<\alpha_{j}\right]$ for $j=1, \ldots, n$, such that the function

$$
\varphi_{A}(x)=u_{0}(x)+\sum_{i=1}^{n} A_{i} x_{i}^{2}
$$

is convex in $\mathbb{R}^{n}$.

The utility of the definition (3.3) is stated in the next two remarks

Remark 3.4. If $u_{0}$ is $\alpha$-semiconvex with constants $\left(A_{1}, \ldots, A_{n}\right)$, then for every $\left(A_{1}^{\prime}, \ldots, A_{n}^{\prime}\right)$, such that $A_{i} \leq A_{i}^{\prime}, i=1, \ldots, n$ the function

$$
\varphi_{A^{\prime}}(x)=u_{0}+\sum_{i=1}^{n} A_{i}^{\prime} x_{i}^{2}
$$

is also convex in $\mathbb{R}^{n}$.

Moreover it follows

Remark 3.5. If $u_{0}$ is strictly $\alpha$-semiconvex with constants $\left(A_{1}, \ldots, A_{n}\right)$ the function

$$
\varphi_{\alpha}(x):=u_{0}(x)+\sum_{i=1}^{n} \alpha_{i} x_{i}^{2},
$$

is strongly convex on $\mathbb{R}^{n}$ with modulus $c=2 \min \left(\alpha_{j}-A_{j}\right)$, because

$$
\varphi_{\alpha}(x)-\frac{c}{2}|x|^{2}=\varphi_{A}+\sum_{i=1}^{n}\left(\alpha_{i}-A_{i}-\frac{c}{2}\right) x_{i}^{2},
$$

is convex on $\mathbb{R}^{n}$. 
We are now interested to state regularity properties of $u$, defined by the semigroup $t \rightarrow Q_{t}$ on the initial datum $u_{0}$ and solution of the Hamilton Jacobi equation (3.3). Our result is suggested by [1].

Theorem 3.6. Let $u_{0}$ strictly $\alpha$-semiconvex, $\alpha$ lower bounded. Then the solution $u(x, t)=\left(Q_{t} u_{0}\right)(x)$ may be written

$$
u(x, t)=K(x, t)-v(x, t)
$$

i.e. as difference of two functions, which are convex, the first one $K \in$ $C^{\infty}\left(\mathbb{R}^{n} \times \mathbb{R}_{+}\right)$, solution of the Hamilton-Jacobi equation (3.3), independent of the initial datum $u_{0}$; the later one $v$ continuous differentiable and its gradient is Lipschitzian with Lipschitz constant $L=\frac{1}{\min _{i}\left(\alpha_{i}-A_{i}\right)}$.

Proof. We begin the proof using the weak convexity

$$
\begin{aligned}
& u(x, t)=\min _{y \in \mathbb{R}^{n}}\left[u_{0}(y)+\sum_{j=1}^{n} \frac{\alpha_{j}}{1-e^{-2 \alpha_{j} t}}\left(y_{j}-e^{-\alpha_{j} t} x_{j}\right)^{2}\right] \\
& =\min _{y \in \mathbb{R}^{n}}\left[\varphi_{\alpha}(y)-\sum_{j=1}^{n} \alpha_{j} y_{j}^{2}+\sum_{j=1}^{n} \frac{\alpha_{j}}{1-e^{-2 \alpha_{j} t}}\left(y_{j}-e^{-\alpha_{j} t} x_{j}\right)^{2}\right] \\
& =\min _{y \in \mathbb{R}^{n}}\left[\varphi_{\alpha}(y)-\sum_{j=1}^{n} \alpha_{j} y_{j}^{2}+\sum_{j=1}^{n} \frac{\alpha_{j}}{1-e^{-2 \alpha_{j} t}}\left(y_{j}^{2}+e^{-2 \alpha_{j} t} x_{j}^{2}-2 e^{-\alpha_{j} t} x_{j} y_{j}\right)\right] \\
& =\sum_{j=1}^{n} \frac{\alpha_{j} e^{-2 \alpha_{j} t}}{1-e^{-2 \alpha_{j} t}} x_{j}^{2}+\min _{y \in \mathbb{R}^{n}}\left[\varphi_{\alpha}(y)+\sum_{j=1}^{n}\left[\frac{\alpha_{j}}{1-e^{-2 \alpha_{j} t}}-\alpha_{j}\right] y_{j}^{2}\right. \\
& \left.-\sum_{j=1}^{n} \frac{2 \alpha_{j} e^{-\alpha_{j} t}}{1-e^{-2 \alpha_{j} t}} x_{j} y_{j}\right]=\sum_{j=1}^{n} \frac{\alpha_{j} e^{-2 \alpha_{j} t}}{1-e^{-2 \alpha_{j} t}} x_{j}^{2} \\
& -\max _{y \in \mathbb{R}^{n}}\left[-\varphi_{\alpha}(y)-\sum_{j=1}^{n} \frac{\alpha_{j} e^{-2 \alpha_{j} t}}{1-e^{-2 \alpha_{j} t}} y_{j}^{2}+\sum_{j=1}^{n} \frac{2 \alpha_{j} e^{-\alpha_{j} t}}{1-e^{-2 \alpha_{j} t}} x_{j} y_{j}\right] \\
& =\sum_{j=1}^{n} \frac{\alpha_{j} e^{-2 \alpha_{j} t}}{1-e^{-2 \alpha_{j} t}} x_{j}^{2}-\max _{y \in \mathbb{R}^{n}}\left[\sum_{j=1}^{n} \frac{2 \alpha_{j} e^{-\alpha_{j} t}}{1-e^{-2 \alpha_{j} t}} x_{j} y_{j}\right. \\
& \left.-\left(\sum_{j=1}^{n} \frac{\alpha_{j} e^{-2 \alpha_{j} t}}{1-e^{-2 \alpha_{j} t}} y_{j}^{2}+\varphi_{\alpha}(y)\right)\right]
\end{aligned}
$$

Define

$$
K(x, t)=\sum_{j=1}^{n} \frac{\alpha_{j} e^{-2 \alpha_{j} t}}{1-e^{-2 \alpha_{j} t}} x_{j}^{2}=\sum_{j=1}^{n} \frac{\alpha_{j}}{e^{2 \alpha_{j} t}-1} x_{j}^{2}
$$

Then

$$
u(x, t)=K(x, t)-\left[\varphi_{\alpha}(\cdot)+K(\cdot, t)\right]^{*}(s),
$$


and

$$
s_{j}=\frac{2 \alpha_{j} e^{-\alpha_{j} t}}{1-e^{-2 \alpha_{j} t}} x_{j} \quad j=1 \ldots n .
$$

The result follows, taking

$$
v(x, t)=\left[\varphi_{\alpha}(\cdot)+K(\cdot, t)\right]^{*}\left(\ldots, \frac{2 \alpha_{j} e^{-\alpha_{j} t}}{1-e^{-2 \alpha_{j} t}} x_{j}, \ldots\right),
$$

observing that $K(x, t) \geq 0$ and $\varphi_{\alpha}$ is strongly convex in $\mathbb{R}^{n}$. Indeed we can apply theorem 4.2.1. Chapter E in [13].

\section{Hypercontractivity and LSI}

\subsection{Weighted hypercontractivity results}

Let us recall that the nonlinear semigroup we are concerned with, is given by

$$
\left(Q_{t} u_{0}\right)(x)=\min _{z}\left\{u_{0}\left(e^{-\alpha t} x+\left(\frac{1-e^{-\alpha p t}}{\alpha p}\right)^{\frac{1}{p}} z\right)+H^{\star}(z)\right\}
$$

and that in paper [4] we get hypercontractivity results, assuming that the initial data $u_{0}$ were admissible, so that

$$
\left\|\exp \left(u_{0}(x)\right)\right\|_{L^{r}\left(R^{n}, d x\right)}<+\infty .
$$

We are dealing here with more general $u_{0}$; we assume that

$$
u_{0}(x)=o\left(H^{\star}(x)\right) \text { for }|x| \rightarrow+\infty .
$$

Then, for positive real number $b$, by (4.1), the following conditions holds

$$
\int_{\mathbb{R}^{n}} \exp \left(r u_{0}(x)-b H^{\star}(x)\right) d x<+\infty .
$$

for every $r \geq 1$. With the assumpion (4.1), in this paper, every Lipschitz function can be taken as initial datum $u_{0}$.

Theorem 4.1. Assume that $u_{0}$ is lsc, $\left(H^{*}, \alpha\right)$ lower bounded function and that $u_{0}$ satisfies (4.1). We fix $1 \leq r \leq s<+\infty$, then there exists a constant $C$, such that

$$
\begin{aligned}
& \left(\int_{\mathbb{R}^{n}} \exp \left[s e^{\alpha t}\left(Q u_{0}\right)(x)-\left(\frac{s}{r}\right)^{q} b H^{\star}(x)\right] d x\right)^{\frac{1}{s e^{\alpha t}}} \\
& \leq C\left(\int_{\mathbb{R}^{n}} \exp \left[r u_{0}(x)-b H^{\star}(x)\right] d x\right)^{\frac{1}{r}}
\end{aligned}
$$

the $C$ constant will be given below. 
Proof. We put: $a=\frac{r}{s} e^{-\alpha t}$, and build the situation in order to apply the Prekopa-Leinder inequality. For positive $\gamma$ (to be fixed later) and $b$, we introduce the functions

$$
\begin{aligned}
u(x) & =\exp \left[s e^{\alpha t} Q_{t} u_{0}(x)-b H^{\star}\left(\frac{s}{r} x\right)\right], \\
v(x) & =\exp \left[-\gamma H^{\star}(x)-b H^{\star}\left(\frac{s}{r} x\right)\right], \\
w(x) & =\exp \left[r u_{0}\left(\frac{s}{r} x\right)-b H^{\star}\left(\frac{s}{r} x\right)\right] .
\end{aligned}
$$

It is easy to get that the functions $u, v$, and $w$ are $L^{1}\left(\mathbb{R}^{N}\right)$, we see that

$$
\begin{aligned}
(u(x))^{a} & =\exp \left[r\left(Q_{t} u_{0}\right)(x)-a b H^{\star}\left(\frac{s}{r} x\right)\right] \\
& \left.\leq \exp \left\{r u_{0}\left(e^{-\alpha t} x+\left(\frac{1-e^{-\alpha p t}}{\alpha p}\right)^{\frac{1}{p}} y\right)+r H^{\star}(y)\right\}-a b H^{\star}\left(\frac{s}{r} x\right)\right\} \\
& =\exp \left\{r u_{0}\left[\frac{s}{r}\left(\frac{r}{s} e^{-\alpha t} x+\frac{r}{s}\left(\frac{1-e^{-\alpha p t}}{\alpha p}\right)^{\frac{1}{p}} y\right)\right]+r H^{\star}(y)-a b H^{\star}\left(\frac{s}{r} x\right)\right\}
\end{aligned}
$$

for all $y \in \mathbb{R}^{n}$. We make a change of variable setting

$$
y=\frac{s}{r}\left(\frac{\alpha p}{1-e^{-\alpha p t}}\right)^{\frac{1}{p}}(1-a) z .
$$

We get

$$
\begin{aligned}
& (u(x))^{a}(v(z))^{1-a} \\
& \leq \exp \left\{r u_{0}\left(\frac{s}{r}(a x+(1-a) z)\right)+r H^{\star}\left(\frac{s}{r}\left(\frac{\alpha p}{1-e^{-\alpha p t}}\right)^{\frac{1}{p}}(1-a) z\right)\right. \\
& \left.\quad-a b H^{\star}\left(\frac{s}{r} x\right)-(1-a) \gamma H^{\star}(z)-(1-a) b H^{\star}\left(\frac{s}{r} z\right)\right\} .
\end{aligned}
$$

We fix $\gamma$ in order to eliminate the second and the fourth terms of the last inequality. It gives

$$
\gamma=r\left(\frac{s}{r}\right)^{q}\left[\frac{\alpha p(1-a)}{1-e^{-\alpha p t}}\right]^{q-1}
$$

Then we get

$$
\begin{aligned}
& (u(x))^{a}(v(z))^{1-a} \\
& \quad \leq \exp \left\{r u_{0}\left(\frac{s}{r}(a x+(1-a) z)\right)-b\left[a H^{\star}\left(\frac{s}{r} x\right)+(1-a) H^{\star}\left(\frac{s}{r} z\right)\right]\right\} \\
& \quad \leq \exp \left\{r u_{0}\left(\frac{s}{r}(a x+(1-a) z)\right)-b H^{\star}\left(\frac{s}{r}(a x+(1-a) z)\right)\right\} \\
& \quad=w(a x+(1-a) z) .
\end{aligned}
$$

Then, by Prekopa-Leinder inequality, we get

$$
\left(\int_{\mathbb{R}^{n}} u(x) d x\right)^{a}\left(\int_{\mathbb{R}^{n}} v(x) d x\right)^{1-a} \leq \int_{\mathbb{R}^{n}} w(x) d x .
$$


To find the constant $C$, we compute

$$
\begin{aligned}
\int_{\mathbb{R}^{n}} w(x) d x & =\int_{\mathbb{R}^{n}} \exp \left[r u_{0}\left(\frac{s}{r} x\right)-b H^{\star}\left(\frac{s}{r} x\right)\right] d x \\
& =\left(\frac{r}{s}\right)^{n} \int_{\mathbb{R}^{n}} \exp \left[r u_{0}(x)-b H^{\star}(x)\right] d x .
\end{aligned}
$$

Furthermore, it results

$$
\begin{aligned}
\int_{\mathbb{R}^{n}} v(x) d x & =\int_{\mathbb{R}^{n}} \exp \left[-\left(\gamma+b\left(\frac{s}{r}\right)^{q}\right) H^{\star}(x)\right] d x \\
& =\left[\gamma+b\left(\frac{s}{r}\right)^{q}\right]^{-\frac{n}{q}} \int_{\mathbb{R}^{n}} \exp \left[-H^{\star}(x)\right] d x
\end{aligned}
$$

Therefore from (4.2), (4.3), (4.4) we get

$$
\begin{aligned}
& \left(\int_{\mathbb{R}^{n}} \exp \left[s e^{\alpha t} Q_{t} u_{0}(x)-b\left(\frac{s}{r}\right)^{q} H^{\star}(x)\right] d x\right)^{\frac{1}{s e^{\alpha t}}} \\
& \leq C\left(\int_{\mathbb{R}^{n}} \exp \left[r u_{0}(x)-b H^{\star}(x)\right] d x\right)^{\frac{1}{r}}
\end{aligned}
$$

and the constant $C$, by (4.3) and (4.4), is given by

$$
\begin{gathered}
C=\left(\int_{\mathbb{R}^{n}} v(x) d x\right)^{-\frac{1-a}{r}}\left(\frac{r}{s}\right)^{\frac{n}{r}} \\
=\left[\left(\frac{s}{r}\right)^{q}\left[b+r\left(\frac{\alpha p(1-a)}{1-e^{-\alpha p t}}\right)^{q-1}\right]\right]^{\frac{n(1-a)}{q r}} \\
\left(\frac{r}{s}\right)^{\frac{n}{r}}\left[\int_{\mathbb{R}^{n}} \exp -H^{\star}(x) d x\right]^{-\frac{1-a}{r}} \\
=\left(\frac{r}{s}\right)^{\frac{n a}{r}}\left[\left(b+r\left(\frac{\alpha p(1-a)}{1-e^{-\alpha p t}}\right)^{q-1}\right)^{\frac{n}{q}}\left(\int_{\mathbb{R}^{n}} \exp \left(-H^{\star}(x)\right) d x\right)^{-1}\right]^{\frac{1-a}{r}} .
\end{gathered}
$$

We may conclude with the condition of hypercontractivity given by

$$
b+r\left(\frac{\alpha p(1-a)}{1-e^{-\alpha p t}}\right)^{q-1} \leq\left(\int_{\mathbb{R}^{n}} \exp \left(-H^{\star}(x)\right) d x\right)^{\frac{q}{n}}, \quad \forall t>0 .
$$

Since $b$ is positive, from (4.5), we obtain the necessary condition

$$
r\left(\frac{\alpha p(1-a)}{1-e^{-\alpha p t}}\right)^{q-1}<\left(\int_{\mathbb{R}^{n}} \exp \left(-H^{\star}(x)\right) d x\right)^{\frac{q}{n}}
$$

and the thesis follows with $C \leq 1$. 


\subsection{A weighted hypercontractivity result.}

The hypercontractivity results on the semigroup $t \rightarrow Q_{t}$ of the paper [3], require the admissibility of the initial datum $u_{0}$ of the Cauchy problem (3.3).

In this paper we state new results, assuming that $u_{0}$ verifies the hypothesis

$$
u_{0}(x)=o\left(|x|^{2}\right) \quad \text { for }|x| \rightarrow+\infty .
$$

Then, by (4.6), for any fixed $b_{j}>0, j=1, \ldots, n$, it results

$$
\int_{\mathbb{R}^{n}} \exp \left[r u_{0}(x)-\sum_{j=1}^{n} b_{j} x_{j}^{2}\right] d x<+\infty,
$$

for every $r \in[1,+\infty)$. As particular case, taking

$$
b_{j}=r \frac{a \alpha_{j}}{1-e^{-2 \alpha_{j} t}}, \quad a=\frac{r}{s} e^{-\beta t},
$$

we also have

$$
\int_{\mathbb{R}^{n}} \exp \left[r u_{0}(x)-\sum_{j=1}^{n} r \frac{a \alpha_{j}}{1-e^{-2 \alpha_{j} t}} x_{j}^{2}\right] d x<+\infty,
$$

for every $r \in[1,+\infty)$.

We are going to describe one of the results of the paper.

Theorem 4.2. We fix $\beta \geq \alpha_{j}, j=1, \ldots, n$, and $1 \leq r \leq s<+\infty, a=\frac{r}{s} e^{-\beta t}$ Assume that $u_{0}$ is a lsc, $\alpha$ lower bounded function and that $u_{0}$ satisfies (4.6). Then there exists a real constan $C$, such that

$$
\begin{aligned}
& \left(\int_{\mathbb{R}^{n}} \exp \left[s e^{\beta t}\left(\left(Q_{t} u_{0}\right)(x)-K(x, t)\right)\right] d x\right)^{\frac{1}{s e^{\beta t}}} \\
& \quad \leq C\left(\int_{\mathbb{R}^{n}} \exp \left[r\left(u_{0}(x)-a L(x, t)\right)\right] d x\right)^{\frac{1}{r}},
\end{aligned}
$$

where

$$
K(x, t)=\sum_{j=1}^{n} \frac{\alpha_{j} e^{-2 \alpha_{j} t}}{1-e^{-2 \alpha_{j} t}} x_{j}^{2} ; \quad L(x, t)=\sum_{j=1}^{n} \frac{\alpha_{j}}{1-e^{-2 \alpha_{j} t}} x_{j}^{2} .
$$

This theorem follows from a more general result, which we give here.

Theorem 4.3. Assume that $u_{0}$ is a lsc, $\alpha$ lower bounded function and that $u_{0}$ satisfies (4.6). We fix $\beta \geq \alpha_{j}, j=1, \ldots, n$, and $1 \leq r \leq s<+\infty, a=\frac{r}{s} e^{-\beta t}$. Then, for every fixed $\xi \in \mathbb{R}^{n}$, there exist $C \in \mathbb{R}_{+}$, depending also by $\xi$, such that

$$
\begin{aligned}
& \left(\int_{\mathbb{R}^{n}} \exp \left[s e^{\beta t}\left(Q_{t} u_{0}\right)(x)-\sum_{j=1}^{n} b_{j}\left(\frac{s}{r} e^{\left(\beta-\alpha_{j}\right) t} x_{j}-\xi_{j}\right)^{2}\right] d x\right)^{\frac{1}{s e^{\beta t}}} \\
& \leq C\left(\int_{\mathbb{R}^{n}} \exp \left[r u_{0}(x)-\sum_{j=1}^{n} b_{j}\left(x_{j}-\xi_{j}\right)^{2}\right] d x\right)^{\frac{1}{r}}
\end{aligned}
$$


Proof. We recall that in this particular case

$$
\left(Q_{t} u_{0}\right)(x)=\min _{y}\left\{u_{0}\left(\ldots, e^{-\alpha_{j} t} x_{j}+\sqrt{1-e^{-2 \alpha_{j} t}} y_{j}, \ldots\right)+\sum_{j=1}^{n} \alpha_{j} y_{j}^{2}\right\} .
$$

Then, for every $y \in \mathbb{R}^{n}$, we have

$$
\left(Q_{t} u_{0}\right)(x) \leq u_{0}\left(\ldots, e^{-\alpha_{j} t} x_{j}+\sqrt{1-e^{-2 \alpha_{j} t}} y_{j}, \ldots\right)+\sum_{j=1}^{n} \alpha_{j} y_{j}^{2} .
$$

In order to apply the Prekopa-Leinder inequality, we introduce the functions

$$
\begin{aligned}
& u(x)=\exp \left[s e^{\beta t} Q_{t} u_{0}(x)-\sum b_{j}\left(\frac{s}{r} e^{\left(\beta-\alpha_{j}\right) t} x_{j}-\xi_{j}\right)^{2}\right], \\
& v(x)=\exp \left[-\sum \gamma_{j} x_{j}^{2}-\sum b_{j}\left(\frac{s}{r} e^{\left(\beta-\alpha_{j}\right) t} x_{j}-\xi_{j}\right)^{2}\right], \\
& w(x)=\exp \left[r u_{0}\left(\ldots, \frac{s}{r} e^{\left(\beta-\alpha_{j}\right) t} x_{j}, \ldots\right)-\sum_{j=1}^{n} b_{j}\left(\frac{s}{r} e^{\left(\beta-\alpha_{j}\right) t} x_{j}-\xi_{j}\right)^{2}\right] .
\end{aligned}
$$

It is easy to get, by (4.6), that the functions $u, v$, and $w$ are $L^{1}\left(\mathbb{R}^{n}\right)$. For positive $\gamma_{j}$ (to be fixed later) and $b_{j}$,

$$
\begin{aligned}
(u(x))^{a} \leq & \exp \left[r u_{0}\left(\ldots, \frac{s}{r} e^{\left(\beta-\alpha_{j}\right) t}\left(\frac{r}{s} e^{-\beta t} x_{j}+\frac{r}{s} e^{-\left(\beta-\alpha_{j}\right) t} \sqrt{1-e^{-2 \alpha_{j} t}} y_{j}\right), \ldots\right)\right. \\
& \left.+r \sum_{j=1}^{n} \alpha_{j} y_{j}^{2}-a \sum_{j=1}^{n} b_{j}\left(\frac{s}{r} e^{\left(\beta-\alpha_{j}\right) t} x_{j}-\xi_{j}\right)^{2}\right] .
\end{aligned}
$$

We make the change of variables

$$
y_{j}=\frac{s e^{\left(\beta-\alpha_{j}\right) t}(1-a)}{r \sqrt{1-e^{-2 \alpha_{j} t}}} z_{j} .
$$

We now compute starting from (4.9)

$$
\begin{aligned}
& (u(x))^{a}(v(z))^{1-a} \leq \exp \left[r u_{0}\left(\ldots, e^{\left(\beta-\alpha_{j}\right) t} \frac{s}{r}\left[a x_{j}+(1-a) z_{j}\right], \ldots\right)\right. \\
& +r \sum_{j=1}^{n} \alpha_{j} \frac{s^{2} e^{2\left(\beta-\alpha_{j}\right) t}(1-a)^{2}}{r^{2}\left(1-e^{-2 \alpha_{j} t}\right)} z_{j}^{2}-a \sum_{j=1}^{n} b_{j}\left(\frac{s}{r} e^{\left(\beta-\alpha_{j}\right) t} x_{j}-\xi_{j}\right)^{2} \\
& \left.-(1-a) \sum_{j=1}^{n} \gamma_{j} z_{j}^{2}-(1-a) \sum_{j=1}^{n} b_{j}\left(\frac{s}{r} e^{\left(\beta-\alpha_{j}\right) t} z_{j}-\xi_{j}\right)^{2}\right] .
\end{aligned}
$$

Here we fix $\gamma_{j}$ as follows

$$
\gamma_{j}=\alpha_{j} \frac{s^{2} e^{2\left(\beta-\alpha_{j}\right) t}(1-a)}{r\left(1-e^{-2 \alpha_{j} t}\right)} .
$$


From (4.10) and the convexity of quadratic function, we get

$$
\begin{aligned}
(u(x))^{a}(v(z))^{1-a} \leq & \exp \left[r u_{0}\left(\ldots, e^{\left(\beta-\alpha_{j}\right) t}\left[a x_{j}+(1-a) z_{j}\right], \ldots\right)\right. \\
& \left.-\sum_{j=1}^{n} b_{j}\left(\frac{s}{r} e^{\left(\beta-\alpha_{j}\right) t}\left[a x_{j}+(1-a) z_{j}\right]-\xi_{j}\right)^{2}\right]
\end{aligned}
$$

i.e.

$$
(u(x))^{a}(v(z))^{1-a} \leq w(a x+(1-a) z) .
$$

By Prekopa-Leinder inequality, (4.11) gives:

$$
\left(\int_{\mathbb{R}^{n}} u(x) d x\right)^{\frac{1}{s e^{\beta t}}} \leq C\left(\int_{\mathbb{R}^{n}} w(x) d x\right)^{\frac{1}{r}} .
$$

Since it is easy to see that

$$
\int_{\mathbb{R}^{n}} w(x) d x=\prod_{j=1}^{n} \frac{r}{s} e^{-\left(\beta-\alpha_{j}\right) t} \int_{\mathbb{R}^{n}} \exp \left[r u_{0}(x)-\sum_{j=1}^{n} b_{j}\left(x_{j}-\xi_{j}\right)^{2}\right],
$$

from (4.12) we obtain the result, with the constant $C$ given by

$$
C=\left(\int_{\mathbb{R}^{n}} v(x) d x\right)^{-\frac{1-a}{r}}\left(\prod_{j=1}^{n} \frac{r}{s} e^{-\left(\beta-\alpha_{j}\right) t}\right)^{\frac{1}{r}}
$$

We are now concerned with the case $\xi=0$. We can obtain explicitely the constant $C$, useful for the hypercontractivity, just computing the integral of the function $v$. Indeed an easy computation gives

$$
v(x)=\exp \left[-\sum_{j=1}^{n} m_{j}^{2} x_{j}^{2}\right]
$$

with

$$
m_{j}=\frac{s}{r} e^{\left(\beta-\alpha_{j}\right) t} \sqrt{(1-a) r \frac{\alpha_{j}}{1-e^{-2 \alpha_{j} t}}+b_{j}} .
$$

It is easy to get

$$
\int_{\mathbb{R}^{n}} v(x) d x=\prod_{j=1}^{n} \int_{\mathbb{R}} e^{-m_{j}^{2} x_{j}^{2}} d x_{j}=\frac{\pi^{\frac{n}{2}}}{\prod_{j=1}^{n} m_{j}} .
$$

It follows from previous results and by some computations

$$
C=\prod_{j=1}^{n}\left\{\left(\frac{r}{s} e^{-\left(\beta-\alpha_{j}\right) t}\right)^{\frac{1}{r}}\left(\frac{1}{\pi}\left[\frac{r(1-a) \alpha_{j}}{1-e^{-2 \alpha_{j} t}}+b_{j}\right]\right)^{\frac{1}{2}\left(\frac{1}{r}-\frac{1}{s e^{\beta t}}\right)}\right\}
$$


It result that for $b_{j}=0, j=1, \ldots, n$, such constant is the same of that given in [3]. We need to investigate for conditions in order to have actual hypercontractivity, i.e. $C \leq 1$. Assume: $s=r$ and $a=e^{-\beta t}$. Since $\beta \geq \alpha_{j}, j=$ $1, \ldots, n$, it seems that the more meaningful conditions are

$$
\frac{r\left(1-e^{-\beta t}\right) \alpha_{j}}{1-e^{-2 \alpha_{j} t}}+b_{j} \leq \pi ; \quad \alpha_{j}<\pi ; \quad \beta \leq 2 \alpha_{j} ; \quad j=1, \ldots, n .
$$

We observe that, if for every $t$ we require the hypercontractivity property, from (4.14), by the positivity of all the $b_{j}$, it necessary to have

$$
r \alpha_{j}<\pi ; \quad \text { for } j=1, \ldots, n \text {. }
$$

We can now get an analogous result to that one obtained in [3].

Theorem 4.4. Under the assumptions

$$
\alpha_{j}<\pi ; \text { and } \max _{j}\left\{\alpha_{j}\right\} \leq \min _{j}\left\{2 \alpha_{j}\right\},
$$

we fix $\beta$ and $b_{j}$ verifying

$$
\max \left\{\alpha_{j}\right\} \leq \beta \leq \min \left\{2 \alpha_{j}\right\} ; 0<b_{j} \leq \frac{1}{2}\left(\pi-\alpha_{j}\right) \quad \text { for } j=1, \ldots, n .
$$

Then, for every function $u_{0}$ which is lsc, $\alpha$ lower bounded, satisfying (4.6), and for every $r$ such that

$$
r \in\left[1, \frac{1}{2}\left(\frac{\pi}{\max \left\{\alpha_{j}\right\}}+1\right)[\right.
$$

the following hypercontractivity property holds true

$$
\left\|\exp \left[Q_{t} u_{0}-\sum_{j=1}^{n} \frac{b_{j}}{r} e^{\left(\beta-2 \alpha_{j}\right) t} x_{j}^{2}\right]\right\|_{L^{r e \beta t}\left(\mathbb{R}^{n}\right)} \leq\left\|\exp \left[u_{0}-\sum_{j=1}^{n} \frac{b_{j}}{r} x_{j}^{2}\right]\right\|_{L^{r}\left(\mathbb{R}^{n}\right)} .
$$

Proof. Thanks to the assumptions the following inequality holds true

$$
b_{j}+r \alpha_{j} \leq \pi \quad \text { for } j=1, \ldots, n .
$$

Then by (4.14) we have $C \leq 1$; then (4.17) follows by the previous result.

\subsection{LSI}

We introduce the functional

$$
G(t)=\left(\int_{\mathbb{R}^{n}} \exp \left[r e^{\beta t}\left(Q_{t} u_{0}\right)(x)-\sum_{j=1}^{n} b_{j} e^{2\left(\beta-\alpha_{j}\right) t} x_{j}^{2}\right] d x\right)^{\frac{1}{r e^{\beta t}}} .
$$

We can state

Theorem 4.5. Assume $u_{0}$ lsc, $\alpha$ lower bounded, and $u_{0}(x)=o\left(|x|^{2}\right)$ as $|x| \rightarrow$ $+\infty$. Furthermore assume (4.15) (4.16). Then the function $G(t)$ is non increasing in the interval $\left[0, t_{\star}[\right.$, with

$$
t_{\star}=\frac{1}{\beta} \ln \frac{1}{2 r_{\star}}\left(\frac{\pi}{\max \left\{\alpha_{j}\right\}}+1\right) .
$$


where

$$
r_{\star} \in\left[1, \frac{1}{2}\left(\frac{\pi}{\max \left\{\alpha_{j}\right\}}+1\right)[\right.
$$

Proof. We fix $0<t_{1}<t_{2}<t_{\star}$. Recalling that (4.17) is true for every $r$ taken in the interval $\left[1, \frac{1}{2}\left(\frac{\pi}{\max \left\{\alpha_{j}\right\}}+1\right)\left[\right.\right.$, we fix $r_{\star}$ in a such interval. We observe that

$$
\begin{aligned}
& r_{\star} e^{\beta t_{1}}<r_{\star} e^{\beta t_{\star}}=\frac{1}{2 r}\left(\frac{\pi}{\max \left\{\alpha_{j}\right\}}+1\right) . \\
& G\left(t_{2}\right)=\left(\int _ { \mathbb { R } ^ { n } } \operatorname { e x p } \left[\left(r_{\star} e^{\beta t_{1}}\right) e^{\beta\left(t_{2}-t_{1}\right)} Q_{t_{2}-t_{1}}\left(Q_{t_{1} u_{0}}\right)(x)\right.\right. \\
& \left.-\sum_{j=1}^{n} b_{j} e^{2\left(\beta-\alpha_{j}\right) t_{1}} e^{2\left(\beta-\alpha_{j}\right)\left(t_{2}-t_{1}\right)} x_{j}^{2}\right)^{\frac{1}{\left.\left(r_{\star} e^{\beta t_{1}}\right) e^{\beta\left(t_{2}-t_{\star}\right.}\right)}}
\end{aligned}
$$

We can apply (4.17) taking $r=r_{\star} e^{\beta t_{1}}, Q_{t_{1}} u_{0}$ as $u_{0}, t=t_{2}-t_{1}$, if it results

$$
A=b_{j} e^{2\left(\beta-\alpha_{j}\right) t_{1}}+r_{\star} e^{\beta t_{1}} \max _{j}\left\{a_{j}\right\} \leq \pi .
$$

To get (4.18) we impose

$$
b_{j} \leq r_{\star} \max _{j}\left\{a_{j}\right\} \frac{\pi-\max _{j}\left\{a_{j}\right\}}{\pi+\max _{j}\left\{a_{j}\right\}} .
$$

Indeed

$$
\begin{aligned}
A & =r_{\star} e^{\beta t_{1}}\left(\frac{b_{j}}{r_{\star}} e^{\left(\beta-2 \alpha_{j}\right) t_{1}}+\max _{j}\left\{a_{j}\right\}\right) \leq r_{\star} e^{\beta t_{1}}\left(\frac{b_{j}}{r_{\star}}+\max _{j}\left\{a_{j}\right\}\right) \\
& \leq \frac{1}{2}\left(\frac{\pi}{\max \left\{\alpha_{j}\right\}}+1\right)\left(\frac{\pi-\max _{j}\left\{a_{j}\right\}}{\pi+\max _{j}\left\{a_{j}\right\}} \max _{j}\left\{a_{j}\right\}+\max _{j}\left\{a_{j}\right\}\right) \\
& =\frac{1}{2}\left(\pi-\max \left\{\alpha_{j}\right\}\right)+\frac{1}{2}\left(\pi+\max \left\{\alpha_{j}\right\}\right)=\pi .
\end{aligned}
$$

Then we apply (4.17) to obtain

$$
G\left(t_{2}\right) \leq G\left(t_{1}\right)
$$

and the thesis follows.

Theorem 4.6. Assume $u_{0}$ strictly $\alpha$-semiconvex, $\alpha$ lower bounded, and $u_{0}(x)=$ $o\left(|x|^{2}\right)$ as $|x| \rightarrow+\infty$. Assume (4.15) (4.16). Fix $r$ such that

$$
r \in\left[1, \frac{1}{2}\left(\frac{\pi}{\max \left\{\alpha_{j}\right\}}+1\right)\right.
$$

Then

$$
\begin{gathered}
\beta E\left(e^{r u_{0}-\sum_{j=1}^{n} b_{j} x_{j}^{2}}\right)+\sum_{i=1}^{n} \alpha_{i} \int_{\mathbb{R}^{n}} e^{r u_{0}-\sum_{j=1}^{n} b_{j} x_{j}^{2}} d x \\
\leq r \int_{\mathbb{R}^{n}} e^{\frac{1}{2} r u_{0}-\sum_{j=1}^{n} b_{j} x_{j}^{2}}\left|D u_{0}(x)\right|^{2} d x+\beta \sum_{i=1}^{n} b_{i} \int_{\mathbb{R}^{n}} e^{r u_{0}-\sum_{j=1}^{n} b_{j} x_{j}^{2}} x_{i}^{2} d x .
\end{gathered}
$$


Proof. We recall the definition of the entropy functional

$$
E(h):=\int_{\mathbb{R}^{n}} h(x) \ln h(x) d x-\int_{\mathbb{R}^{n}} h(x) d x \ln \int_{\mathbb{R}^{n}} h(x) d x
$$

We introduce the function

$$
g(x)=\left[r e^{\beta t}\left(Q_{t} u_{0}\right)(x)-\sum b_{j} e^{2\left(\beta-\alpha_{j}\right) t} x_{j}^{2}\right],
$$

with

$$
G(t)=\left(\int_{\mathbb{R}^{n}} \exp \left[r e^{\beta t}\left(Q_{t} u_{0}\right)(x)-\sum_{j=1}^{n} b_{j} e^{2\left(\beta-\alpha_{j}\right) t} x_{j}^{2}\right] d x\right)^{\frac{1}{r e^{\beta t}}} .
$$

We consider $G(t)^{r e^{\beta t}}$, we make its derivative, and, using $G^{\prime}(t) \leq 0$ for every $t \in\left(0, t_{\star}\right)$ (with the notation of the previous theorem), we get

$$
\begin{aligned}
& \beta E\left(e^{g}\right)+\sum_{j=1}^{n} \alpha_{j} \int_{\mathbb{R}^{n}} e^{g(x)} d x \\
& \leq r e^{\beta t} \int_{\mathbb{R}^{n}} \exp \left(\frac{1}{2} g(x)\right)\left|D Q_{t} u_{0}(x)\right|^{2} d x+\beta \int_{\mathbb{R}^{n}} e^{g(x)}\left(\sum_{j=1}^{n} b_{j} e^{2\left(\beta-\alpha_{j}\right) t} x_{j}^{2}\right) d x .
\end{aligned}
$$

The result follows as $t$ goes to $0^{+}$.

\section{LSI from general hypercontractivity}

In all this section we assume $D H \in C_{l o c}^{0,1}\left(\mathbb{R}^{n}\right)$

Theorem 5.1. Assume $u_{0} l s c,\left(H^{\star}, \alpha\right)$ lower bounded and $u_{0}(x)=o\left(H^{\star}(x)\right)$ as $|x| \rightarrow+\infty$. Assume

$$
(p \alpha)^{q-1}<\left(\int_{\mathbb{R}^{n}} \exp \left(-H^{\star}(x)\right) d x\right)^{\frac{q}{n}}=: B,
$$

and take

$$
b=\frac{1}{2}\left(B-(p \alpha)^{q-1}\right) .
$$

Then, for any fixed

$$
\begin{aligned}
& r \in\left[1, \frac{1}{2}\left(\frac{B}{(p \alpha)^{q-1}}+1\right)[\right. \\
& \left(\int_{\mathbb{R}^{n}} \exp \left[r e^{\alpha t}\left(Q_{t} u_{0}(x)\right)-b H^{\star}(x)\right] d x\right)^{\frac{1}{r e^{\alpha t}}} \leq\left(\int_{\mathbb{R}^{n}} \exp \left[r u_{0}(x)-b H^{\star}(x)\right] d x\right)^{\frac{1}{r}}
\end{aligned}
$$

Proof. By the assumptions

$$
b+r(\alpha p)^{q-1} \leq\left(\int_{\mathbb{R}^{n}} \exp \left(-H^{\star}(x) d x\right)^{\frac{q}{n}},\right.
$$


and using that

$$
\psi(t)=\frac{1-e^{-\alpha t}}{1-e^{-\alpha p t}},
$$

is an increasing function on the positive real, we have that the hypercontractivity condition

$$
b+r\left(\frac{\alpha p\left(1-e^{-\alpha t}\right)}{1-e^{-\alpha p t}}\right)^{q-1} \leq\left(\int_{\mathbb{R}^{n}} \exp \left(-H^{\star}(x)\right) d x\right)^{\frac{q}{n}}
$$

is satisfied, hence the result follows.

We define the function

$$
G_{H}(t)=\left(\int_{\mathbb{R}^{n}} \exp \left(r e^{\alpha t} Q_{t} u_{0}(x)-b H^{\star}(x) d x\right)\right)^{\frac{1}{r e^{\alpha t}}},
$$

with a fixed $r$ in the set

$$
\left[1, \frac{1}{2}\left(\frac{B}{(p \alpha)^{q-1}}+1\right)\right)
$$

and we set

$$
t_{\star}=\frac{1}{\alpha} \ln \left(\frac{1}{2 r}\left(\frac{B}{(p \alpha)^{q-1}}+1\right)\right) .
$$

We recall the definition of the entropy function with respect to

$$
\begin{aligned}
& d \Gamma=\exp \left(-b H^{\star}\right) d x \\
& \mathcal{E}(h, d \Gamma):=\int_{\mathbb{R}^{n}} h(x) \ln h(x) d \Gamma-\int_{\mathbb{R}^{n}} h(x) d \Gamma \ln \int_{\mathbb{R}^{n}} h(x) d \Gamma
\end{aligned}
$$

Theorem 5.2. Assume $u_{0}$ lsc and $\left(H^{\star}, \alpha\right)$ lower bounded and $u_{0}(x)=o\left(H^{\star}(x)\right)$ as $|x| \rightarrow+\infty$. Assume

$$
(p \alpha)^{q-1}<\left(\int_{\mathbb{R}^{n}} \exp \left(-H^{\star}(x) d x\right)^{\frac{q}{n}},\right.
$$

then the function $G_{H}(t)$, defined by $(5.3)$, is non increasing in $\left(0, t_{\star}\right)$.

Proof. We fix $0<t_{1}<t_{2}<t_{\star}$.

$G_{H}\left(t_{2}\right)=\left(\int_{\mathbb{R}^{n}} \exp \left(r e^{\alpha t_{1}} e^{\alpha\left(t_{2}-t_{1}\right)} Q_{t_{2}-t_{1}} Q_{t_{1}} u_{0}(x)-b H^{\star}(x) d x\right)\right)^{\frac{1}{r e^{\alpha t_{1}} e^{\alpha\left(t_{2}-t_{1}\right)}}}$.

On the other hand, since $t_{1}<t_{\star}$,

$$
r e^{\alpha t_{1}}<r^{\alpha t_{\star}}=\frac{1}{2}\left(\frac{B}{(p \alpha)^{q-1}}+1\right)
$$

We can apply (5.1) taking $r=r_{\star} e^{\beta t_{1}}, Q_{t_{1}} u_{0}$ as $u_{0}, t=t_{2}-t_{1}$, and the thesis follows. 
Theorem 5.3. To simplify, we assume $u_{0} \in C^{1,1}\left(\mathbb{R}^{n}\right),\left(H^{\star}, \alpha\right)$ bounded and $u_{0}(x)=o\left(H^{\star}(x)\right)$ as $|x| \rightarrow+\infty$. Assume

$$
(p \alpha)^{q-1}<\left(\int_{\mathbb{R}^{n}} \exp \left(-H^{\star}(x)\right) d x\right)^{\frac{q}{n}},
$$

fix

$$
b=\frac{1}{2}\left(\left(\int_{\mathbb{R}^{n}} \exp \left(-H^{\star}(x)\right) d x\right)^{\frac{q}{n}}-(p \alpha)^{q-1}\right),
$$

and

$$
r \in\left[1, \frac{1}{2}\left(\frac{\left(\int_{\mathbb{R}^{n}} \exp \left(-H^{\star}(x)\right) d x\right)^{\frac{q}{n}}}{(p \alpha)^{q-1}}+1\right)\right) .
$$

Then the following LSI holds

$$
\begin{aligned}
& \alpha \mathcal{E}\left(e^{r u_{0}(x)} ; d \Gamma\right)+\alpha n \int_{\mathbb{R}^{n}} e^{r u_{0}(x)} d \Gamma \\
& \quad \leq \frac{p^{p}}{r^{p-1}} \int_{\mathbb{R}^{n}} H\left(D e^{\frac{r}{p}} u_{0}(x)\right) d \Gamma+\alpha q b \int_{\mathbb{R}^{n}} e^{r u_{0}(x)} H^{\star}(x) d \Gamma
\end{aligned}
$$

Proof. We consider

$$
\left(G_{H}(t)\right)^{r e^{\alpha t}}=\int_{\mathbb{R}^{n}} \exp \left(r e^{\alpha t} Q_{t} u_{0}(x)-b H^{\star}(x) d x .\right.
$$

Making the derivative, we get

$$
\begin{gathered}
\left.r e^{\alpha t}\left(G_{H}(t)\right)^{r e^{\alpha t}-1} G_{H}^{\prime}(t)\right)=-\alpha\left(G_{H}(t)\right)^{r e^{\alpha t}} \ln \left(G_{H}(t)\right)^{r e^{\alpha t}} \\
+\int_{\mathbb{R}^{n}} \exp \left(r e^{\alpha t} Q_{t} u_{0}(x)-b H^{\star}(x)\right) \alpha r e^{\alpha t} Q_{t} u_{0}(x) d x \\
+\int_{\mathbb{R}^{n}} \exp \left(r e^{\alpha t} Q_{t} u_{0}(x)-b H^{\star}(x)\right) r e^{\alpha t} \partial_{t} Q_{t} u_{0}(x) d x
\end{gathered}
$$

By $G_{H}^{\prime}(t) \leq 0$ and by the definition of $\mathcal{E}$ it results

$$
\begin{aligned}
\alpha \mathcal{E} & \left.\left(\exp \left(r e^{\alpha t} Q_{t} u_{0}(x)\right) ; d \Gamma\right)\right) \\
\leq & \int_{\mathbb{R}^{n}} \exp \left(r e^{\alpha t} Q_{t} u_{0}(x)\right) r e^{\alpha t}\left[\alpha x\left(D Q_{t} u_{0}\right)(x)+H\left(D Q_{t} u_{0}\right)(x)\right] d \Gamma \\
= & \alpha \int_{\mathbb{R}^{n}}\left\{D \exp \left(r e^{\alpha t} Q_{t} u_{0}(x)\right)\right\} x d \Gamma \\
& +\frac{r e^{\alpha t} \quad p}{\left(\frac{r}{p} e^{\alpha t}\right)} \int_{\mathbb{R}^{n}} H\left(D \exp \left(\frac{r}{p} e^{\alpha t} Q_{t} u_{0}(x)\right) d \Gamma\right. \\
= & -\alpha n \int_{\mathbb{R}^{n}} \exp \left(r e^{\alpha t} Q_{t} u_{0}(x)\right) d \Gamma \\
& -\alpha \int_{\mathbb{R}^{n}} \exp \left(r e^{\alpha t} Q_{t} u_{0}(x)\right)\left(x D\left(-b H^{\star}(x)\right) d \Gamma\right.
\end{aligned}
$$




$$
+\frac{r e^{\alpha t}}{\left(\frac{r}{p} e^{\alpha t}\right)} \int_{\mathbb{R}^{n}} H\left(D \exp \left(\frac{r}{p} e^{\alpha t} Q_{t} u_{0}(x)\right)\right) d \Gamma
$$

Since

$$
\begin{aligned}
& x D\left(H^{\star}(x)\right)=q H^{\star}(x) \\
& \left.\alpha \mathcal{E}\left(\exp \left(r e^{\alpha t} Q_{t} u_{0}(x)\right) ; d \Gamma\right)\right)+\alpha n \int_{\mathbb{R}^{n}} \exp \left(r e^{\alpha t} Q_{t} u_{0}(x)\right) d \Gamma \\
& \quad \leq \frac{p^{p}}{r^{p-1} e^{\alpha(p-1) t}} \int_{\mathbb{R}^{n}} H\left(D e^{\frac{r}{p} e^{\alpha t} Q_{t} u_{0}}\right) d \Gamma+\alpha q b \int_{\mathbb{R}^{n}} e^{r e^{\alpha t}\left(Q_{t} u_{0}\right)(x)} H^{\star}(x) d \Gamma
\end{aligned}
$$

The result follows sending $t \rightarrow 0^{+}$.

\section{Appendix}

Here we show some properties of the semigroup $t \rightarrow Q_{t}$ helpful to get LSI inequalities in the limit as $t \rightarrow 0^{+}$in (5.4).

Proposition 6.1. Assume $D H, D u_{0} \in C_{l o c}^{0,1}\left(\mathbb{R}^{n}\right)$, then for any $R \in(0,+\infty)$ there exists $t_{0} \in(0,+\infty)$ such that for any $x \in B_{R}^{0}=\left\{z \in \mathbb{R}^{n}:|z|<R\right\}, t \in$ $\left(0, t_{0}\right]$ there exists a unique $y=y(x, t) \in B_{R}^{0}$ such that

$$
Q_{t} u_{0}(x)=u_{0}(y)+\left(\frac{\alpha p}{1-e^{-\alpha p t}}\right)^{q-1} H^{\star}\left(y-e^{-\alpha t} x\right) .
$$

Furthermore

$$
\lim _{t \rightarrow 0^{+}} y(x, t)=x, \quad \text { uniformly in } B_{R} .
$$

Proof. Let $L_{H}$ and $L_{0}$ the local Lipschitz constants of, respectively, $D H, D u_{0}$, then we set

$$
M=\max \left\{\left|D H\left(D u_{0}(y)\right)\right| y \in B_{R}\right\} .
$$

To find $y$ we need to solve the equation

$$
D u_{0}(y)+D H^{\star}\left(\left(\frac{\alpha p}{1-e^{-\alpha p t}}\right)\left(y-e^{-\alpha t} x\right)\right)=0,
$$

equivalent to

$$
y=e^{-\alpha t} x-\left(\frac{1-e^{-\alpha p t}}{\alpha p}\right) D H\left(D u_{0}(y)\right)
$$

We fix $t_{1}$ and $t_{2}$ in $(0,+\infty)$ such that

$$
\begin{gathered}
M\left(\frac{1-e^{-\alpha p t_{1}}}{\alpha p}\right)<R \\
\left(\frac{1-e^{-\alpha p t_{2}}}{\alpha p}\right) L_{H} L_{0}<1
\end{gathered}
$$


Set

$$
t_{0}=\min \left\{t_{1}, t_{2}\right\}
$$

The application

$$
y \rightarrow e^{-\alpha t} x-\left(\frac{1-e^{-\alpha p t}}{\alpha p}\right) D H\left(D u_{0}(y)\right) \quad t \in\left[0, t_{0}\right]
$$

is a contraction. Indeed consider the iterative sequence

$$
\left\{\begin{array}{l}
y_{n+1}=e^{-\alpha t} x-\left(\frac{1-e^{-\alpha p t}}{\alpha p}\right) D H\left(D u_{0}\left(y_{n}\right)\right), \quad n \in \mathbb{N} \\
y_{0}=e^{-\alpha t} x
\end{array}\right.
$$

then

$$
\left|y_{n+1}-y_{n}\right| \leq\left(\frac{1-e^{-\alpha p t}}{\alpha p}\right) L_{H} L_{0}\left|y_{n}-y_{n-1}\right|<\left(\frac{1-e^{-\alpha p t}}{\alpha p}\right)^{n+1}\left(L_{H} L_{0}\right)^{n} M
$$

From this we have the existence and uniqueness of $y$ and

$$
\lim _{t \rightarrow 0^{+}} y_{n}(x, t)=x, \quad \forall n \in \mathbb{N},
$$

uniformly in $B_{R} \times\left[0, t_{0}\right]$.

Theorem 6.2. Assume $D H, D u_{0} \in C_{l o c}^{0,1}\left(\mathbb{R}^{n}\right), y=y(x, t)$ as in proposition (6.1), then for any $(x, t) \in B_{R} \times\left[0, t_{0}\right]$

$$
D\left(Q_{t} u_{0}\right)=e^{-\alpha t} D u_{0}(y(x, t))
$$

and, passing to the limit,

$$
\left.\lim _{t \rightarrow 0^{+}} D\left(Q_{t} u_{0}\right)=\lim _{t \rightarrow 0^{+}} D u_{0}(y(x, t))=D u_{0}(x)\right)
$$

Proof. Set

$$
w(x, t)=y(x, t)-e^{-\alpha t} x .
$$

Then

$$
\begin{gathered}
Q_{t} u_{0}\left(x_{1}\right)-Q_{t} u_{0}(x) \leq u_{0}\left(e^{-\alpha t} x_{1}+w(x, t)\right)-u_{0}\left(e^{-\alpha t} x+w(x, t)\right) \\
Q_{t} u_{0}\left(x_{1}\right)-Q_{t} u_{0}(x) \geq u_{0}\left(e^{-\alpha t} x_{1}+w\left(x_{1}, t\right)\right)-u_{0}\left(e^{-\alpha t} x+w\left(x_{1}, t\right)\right) .
\end{gathered}
$$

Let $e_{i}=\left(\delta_{1 i}, \ldots, \delta_{n i}\right)$ and $h>0$ we consider

$$
x_{1}=x+h e_{i} .
$$

By inequality (6.2) and (6.3) the following limit exists and it results

$$
\lim _{h \rightarrow 0^{+}} \frac{Q_{t} u_{0}\left(x_{1}\right)-Q_{t} u_{0}(x)}{h}=e^{-\alpha t} \partial_{x_{i}} u_{0}\left(e^{-\alpha t} x+w(x, t)\right)
$$

A similar argument applies to negative $h$. Hence the result follows. 


\section{References}

[1] Attouch, H., Aze, D.: Approximation and regularization of arbitrary funtions in Hilbert spaces by the Lasry-Lions method. Annales de l'I.H.P., Section C, Tome 10(3), 289-312 (1993)

[2] Avantaggiati, A., Loreti, P.: Hypercontractivity, Hopf-Lax type formulas, Ornstein-Uhlenbeck operators (I). In: Giga, G., Ishii, K., Koike, S., Ozawa, T., Yamada, N. (eds.) International Conference for the 25th Anniversary of Viscosity Solutions. GAKUTO International Series Mathematical Sciences and Applications, vol. 30, pp. 15-31, (2008)

[3] Avantaggiati, A., Loreti, P.: Hypercontractivity, Hopf-Lax type formulas, Ornstein-Uhlenbeck operators. II. Discrete Contin. Dyn. Syst. Ser. S 2(3), 525-545 (2009)

[4] Avantaggiati, A., Loreti, P.: Hopf-Lax type formulas and hypercontractivity. Ric. Mat. 57(2):171-202 (2008)

[5] Bobkov, S.G., Gentil, I., Ledoux, M.: Hypercontractivity of Hamilton-Jacobi equations. J. Math. Pures Appl.(9) 80(7), 669-696 (2001)

[6] Cannarsa, P., Sinestrari, C.: Semiconcave functions, Hamilton-Jacobi equations, and optimal control. BirkhŠuser, Boston, MA 58 (2004)

[7] Crandall, M.G., Evans, L.C., Lions, P.-L.: Some properties of viscosity solutions of Hamilton-Jacobi equations. Trans. Am. Math. Soc. 282(2), 487-502 (1984)

[8] Evans, L.C.: Partial Differential Equations. In: Graduate Studies in Mathematics. American Mathematical Society (1998)

[9] Ishii, H.: Hopf-Lax formulas for Hamilton-Jacobi equations with semicontinuous initial data. Singularity theory and differential equations (Kyoto, 1999). Surikaisekikenkyusho Kokyuroku No. 1111, pp. 144-156 (1999)

[10] Alvarez, O., Barron, E.N., Ishii, H.: Hopf-Lax formulas for semicontinuous data. Indiana Univ. Math. J. 48(3), 993-1035 (1999)

[11] Gentil, I.: Ultracontractive bounds on Hamilton-Jacobi solutions. Bull. Sci. Math. 126(6), 507-524 (2002)

[12] Gentil, I.: The general optimal $L^{p}$-Euclidean logarithmic Sobolev inequality by Hamilton-Jacobi equations. J. Funct. Anal. 202, 591-599 (2003)

[13] Hiriart-Urruty, J.-B., LemarŽchal, C.: Fundamentals of convex analysis. Abridged version of Convex analysis and minimization algorithms. I Springer, Berlin (1993) (and II. Grundlehren Text Editions. Springer, Berlin, 2001)

[14] Otto, F., Villani, C.: Comment on: Hypercontractivity of Hamilton-Jacobi equations [J. Math. Pures Appl. (9) 80 (2001), no. 7, 669-696] by S.G. Bobkov, I. Gentil and M. Ledoux. J. Math. Pures Appl. (9) 807, 697-700 (2001) 
Antonio Avantaggiati

Dipartimento di Scienze di Base e Applicate per l'Ingegneria Sapienza Università di Roma

via A. Scarpa 16

00161 Rome

Italy

Paola Loreti

Dipartimento di Scienze di Base e Applicate per l'Ingegneria Sezione di Matematica

Sapienza Università di Roma

via A. Scarpa 16

00161 Rome

Italy

e-mail: loreti@dmmm.uniroma1.it

Received: 18 December 2010.

Accepted: 11 February 2012. 